\title{
Staging Surgery for The Treatment of Aortic Arch Aneurysm Combined with Aberrant Bilateral Subclavian Artery, Persistent Left Superior Vena Cava and Airway Compression: A Case Report
}

\author{
Jiayu Fu, MS, Chunjian Shen, MD, Shun Gao, BS, Chao Liu, BS, Wei Liu, MS, Yang Nan, MS, \\ Sheng Qu, MS, Wenzhe Wu, MS, Yao Ge, MS, Xiaonan Lv, MS \\ Department of Cardio-Thoracic Surgery, Second Affiliated Hospital of Shenyang Medical College
}

\section{ABSTRACT}

Background: To describe staging surgery for the treatment of a patient with aortic arch aneurysm combined with aberrant bilateral subclavian artery, persistent left superior vena cava (PLSVC), and airway compression.

Case report: A 42-year-old female was hospitalized for aortic arch aneurysm involving aberrant bilateral subclavian artery, PLSVC, and airway compression. The patient's aneurysm was successfully treated by stage I surgery, including total aortic arch replacement and stented elephant trunk procedure and stage II surgery, including tracheal stenting and tracheotomy. Aortic CTA examination showed an unobstructed lumen and a good stent position without tracheal stent migration. Regular postoperative follow-up showed no complications, such as dyspnea, cough, and sputum, or other discomfort symptoms.

Conclusions: Total aortic arch replacement, elephant trunk surgery, and second-stage tracheal stent surgery are effective and safe for the treatment of aortic arch aneurysm combined with aberrant bilateral subclavian artery, PLSVC, and airway compression.

\section{INTRODUCTION}

Aortic aneurysm is a cardiovascular disease with high mortality and poor natural prognosis. The 5-year survival rate of non-surgical intervention is only $20 \%$ [Chau 2013]. Aortic arch aneurysm, as one type of aortic aneurysm,

Received fune 17, 2019; received in revised form August 19, 2019; accepted August 21, 2019.

The study was supported by Liaoning Provincial Key R\&D Guidance Project Item (No. 20197H8/10300048), Shenyang Science and Technology Plan Project (No. 19-112-4-042) and Shenyang Medical College Science and Technology Fund Project (No. 20192045, No. 20192054 and No. 20192060).

Correspondence: Dr: Chunjian Shen, Department of Cardio-Thoracic Surgery, Second Affiliated Hospital of Shenyang Medical College, No. 64 Qishan Road, Huanggu District, Shenyang City, Liaoning Province, 110035, China; +8618602426455 (e-mail: shenchunjian1976@hotmail.com). involves 3 significant vessels on the arch (innominate artery, left common carotid artery, and left subclavian artery), due to its special anatomic site. Therefore, the operation is complicated and challenging, with a high incidence of intraoperative and postoperative mortality and neurological complications. Open surgery, combined with endovascular repair, has become a potential treatment for the reconstruction of the aortic arch in the world [Kim 2009].

Aortic aneurysm has a variety of clinical manifestations, which involve compression of adjacent organs, especially the upper airway compression. Symptoms of aortic aneurysm-related tracheal compression include dyspnea, cough, expectoration, and even pneumonia [Sabra 2006; Arima 2002]. Although tracheal compression is not fatal, surgery is still the effective treatment of choice [Xiong 2013]. Vascular variation is a significant challenge in cardiac surgery. Persistent left superior vena cava (PLSVC) combined with a variety of bilateral subclavian artery originates from the aortic arch and increases the risk of surgery.

In this study, we described a case of aortic arch aneurysm combined with PLSVC and upper airway compression. This patient was treated with stage I surgery of total aortic arch replacement and elephant trunk surgery and stage II surgery of tracheal stenting. The procedure produced good clinical results, which are summarized in this report.
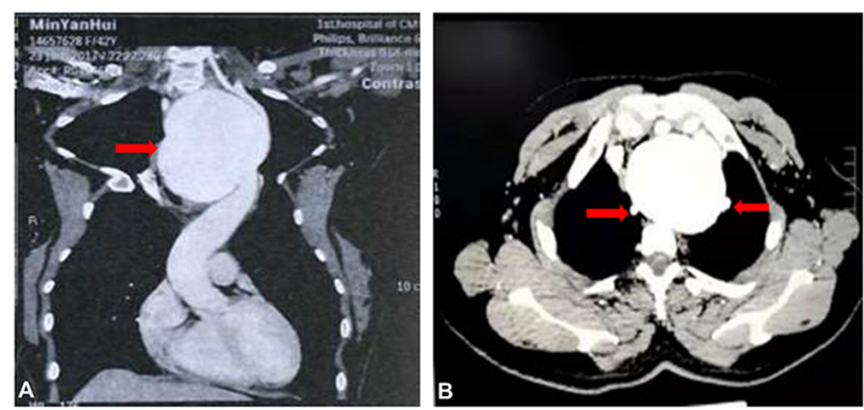

Figure 1. Preoperative aortic CTA images. A). The arrow indicates an aortic aneurysm with a maximum transverse diameter of about $8.3 \mathrm{~cm}$ in the aortic area. B). The arrows indicate that the bilateral subclavian artery originates from the descending aorta. 

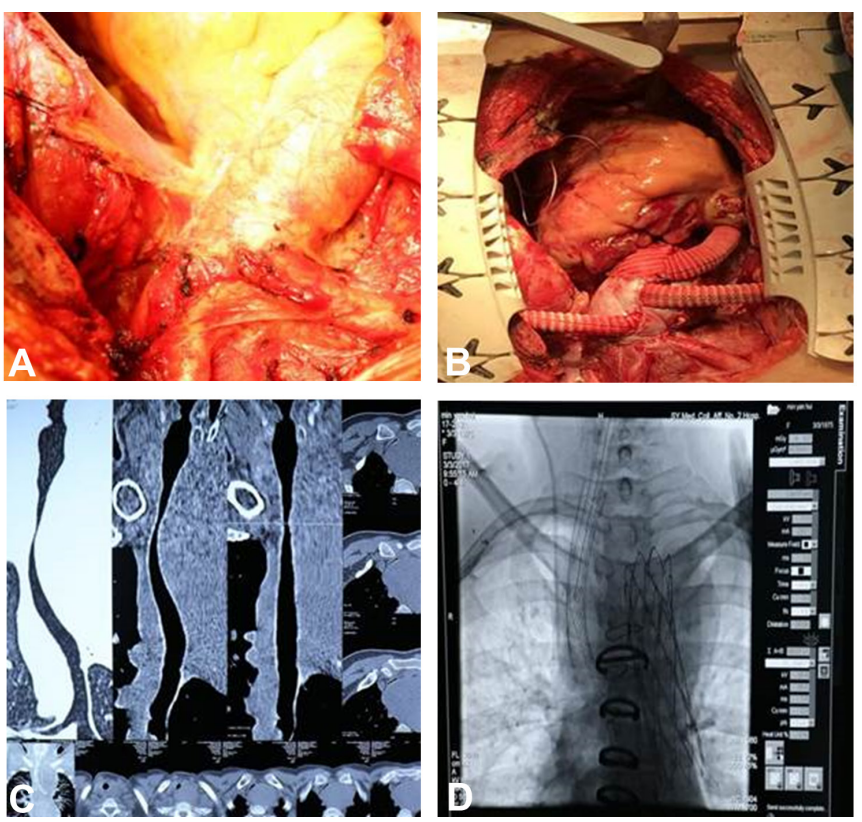

Figure 2. Intraoperative images. A). The origin of the aortic aneurysm and the bilateral subclavian artery. B). Postoperative image after total aortic arch replacement and stented elephant trunk procedure combined with descending aortic arch. C). The position and degree of tracheal stenosis. D) The position of the endovascular stent.

\section{CASE REPORT}

A 42-year-old female with a history of previous aortic arch aneurysm for 4 years presented with dyspnea and cough for 1 month and was admitted to our hospital. Computed tomography angiography (CTA) showed visible distension of lumens of the aortic arch and descending aorta, with a maximum transverse diameter of about $8.3 \mathrm{~cm}$ and a length of about $14.2 \mathrm{~cm}$. The left common carotid artery was locally compressed, and the lumen of the brachiocephalic trunk was slender (Figure 1A and 1B). CT imaging of the lungs showed compressed surrounding tissues and widening of the descending part of the aortic arch with a maximum diameter of about $77 \mathrm{~mm}$. The trachea was compressed and deflected to the right front, and the left trachea was slightly compressed at the bifurcation. The right arteria subclavia was derived directly from the aorta, and the bilateral arteria subclavia outlet was located behind the aortic arch. Interstitial inflammation changes were found in the right lung.

According to the patient's symptoms and imaging findings, two operations (stage I and stage II surgery) were performed. Stage I surgery included total aortic arch replacement and stented elephant trunk procedure combined with descending aortic arch. Stage II surgery included tracheal stenting and tracheotomy. Preoperative echocardiography showed no intracardiac lesions, and routine examination revealed no apparent contraindications.

For stage I surgery, the bilateral axillary arteries and right femoral artery were surgically exposed after general anesthesia with the patient in the supine position. Exploratory
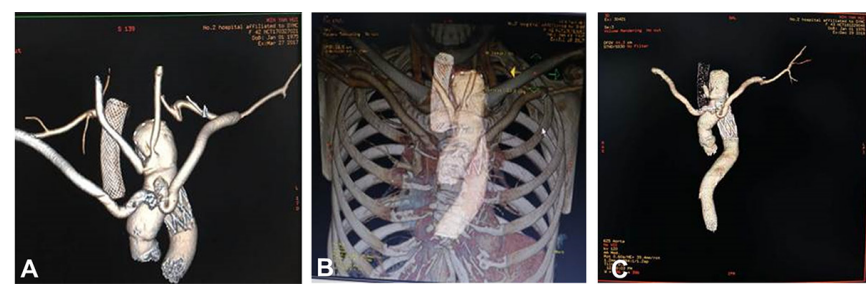

Figure 3. Review of aortic CTA examination at postoperative A) 3 months, B) 6 months, and C) 2 years showed that the reconstructed vascular lumen was unobstructed, the position of the endovascular stent was good.

thoracotomy was then performed. Abnormal ascending aorta was observed, and the bilateral subclavian artery aberrate to the descending aorta of which ostium was located at the aneurysm body. The aneurysm was found in the descending part of the aortic arch with a maximum diameter of $8.0 \mathrm{~cm}$. The proximal ends of the aneurysmal neck were located at the ostium of the left common carotid artery, and the persistent left superior vena cava (PLSVC) can be observed (Figure 2A). The left and right common carotid arteries were dissociated and cannulated. Systemic heparinization was performed to achieve anticoagulation. Extracorporeal circulation was then established via the right femoral artery and the right auricle cannulation.

The left ventricular decompression catheter was inserted into the right upper pulmonary veins. During collateral circulation, the cardioplegia cannula was inserted into the aortic root. The ascending aorta was then clamped; the cold blood cardioplegia was perfused into the aortic root. The heart was protected during arrest with sterile saline ice slush. When the nasopharyngeal temperature decreased to $21^{\circ} \mathrm{C}$, the left common carotid artery was blocked. The aorta was dissected after the circulation arrest. Then the right common carotid artery was cannulated, and cerebral perfusion performed to prevent femoral artery cannulation. The ostium of the left and right subclavian artery within the descending aortic lumen were sutured and delivered into the trunk stent (Intraoperative stent, MicroPort Endovascular (Shanghai) Co., Ltd., 26mm*120mm) through aorta incision. The proximal end of the left and right subclavian artery was set at the ostium of the left common carotid artery. Island anastomosis was performed between the left and right common carotid arteries and the proximal end of the intraoperative stent. Then the distal end of the 4-branched vascular graft (Maquet (Suzhou) Co., Ltd., $24 * 10^{*} 8^{*} 8^{*} 10 \mathrm{~mm}$ ) was anastomosed to the proximal aorta of the intraoperative stent. Subsequently, the cerebral perfusion was stopped; the femoral artery was opened. The proximal end of the vascular graft was anastomosed to the ascending aorta on the coronary sinus. After anastomosis was completed, the ascending aorta was opened, and the heart was re-jumped. The vascular grafts were wrapped with part of the pericardium and the dissected aortic wall, and then the right atrial shunt was conducted. After shunt operation, a subcutaneous tunnel was created between the right and left second intercostal spaces. The $8 \mathrm{~mm}$ branch blood vessels passed through the subcutaneous tunnel and were anastomosed to the left and right axillary arteries, respectively. 
The heart rate of the pacemaker was adjusted to 100 beats/ min, and the circulation was resumed (Figure 2B). The operation was uneventful, with no evidence of adverse effects. The patient returned to the intensive care unit (ICU) for care. Tracheal angiography 7 days postoperatively showed a $40 \%$ stenosis in the upper part of the trachea and a $20 \%$ stenosis in the middle part of the trachea. On the same day, offline extubation was performed, but acute dyspnea occurred 2 hours later. 3D-CT imaging showed severe stenosis of the main trachea (Figure 2C). Then, trachea cannula and assisted ventilation with ventilators were performed again.

For stage II surgery, tracheal stenting and tracheotomy were performed 9 days after stage I surgery. After routine anesthesia, a $5 \mathrm{~F}$ cobra catheter was advanced into the left main bronchus via trachea cannula, the catheter slowly was withdrawn, and the contrast agent was then injected. High degree stenosis of the middle segment of the left main bronchus was observed. Then stiffening long guidewire was deployed, the catheter and trachea cannula were pulled out. Subsequently, after the delivery and release of the stent (Figure 2D), routine tracheotomy was performed. Postoperative aortic CTA examination showed unobstructed reconstructed vascular lumen and a good stent position with no tracheal stent migration (Figure 3A).

Regular postoperative follow-up showed that no complications, such as dyspnea, cough, and sputum, or other discomfort symptoms. The patient's quality of life was significantly improved. Aortic CTA examination showed an unobstructed lumen and a good stent position without no migration (Figure $3 \mathrm{~A}$ and $3 \mathrm{~B})$.

\section{DISCUSSION}

Aortic arch aneurysms account for approximately $15 \%$ of thoracic aortic aneurysms [Bickerstaff 1982]. Aortic arch aneurysm can be found by chest X-Ray, echocardiography, and CT examination since approximately $50 \%$ of patients have no obvious symptoms [Khalil 2007]. Aortic aneurysms involving the descending part of the aortic arch usually are treated with a two-stage procedure [Crawford 1984; Crawford 1989]. This case is an example of the successful usage of total aortic arch replacement, elephant trunk surgery, and second-stage tracheal stent surgery for the treatment of aortic arch aneurysm combined with aberrant bilateral subclavian artery, PLSVC and airway compression.

In 1983, Borst et al. used an elephant trunk to introduce a new method for stage treatment of complex aortic diseases, which simplified the difficulty of staging surgery and achieved better therapeutic results [Borst 1983]. In 1996, Kato et al. began to use the concept of stented elephant trunk procedure [Kato 1996; Kato 2002]. The stented elephant trunk procedure used in this case combined the idea of elephant trunk and stent vessels, which could be performed through a median sternal incision. The main advantage of a stented elephant trunk procedure is that it can be anchored to the wall of the descending aortic aneurysm and promote thrombosis between the blood vessels and the aneurysm wall, thereby avoiding the possibility of reoperation due to advanced neoplasia and rupture of the descending aorta.

PLSVC is relatively rare, with an incidence of about $0.3 \%$ in the population [Chokr 2018], and the clinical detection rate is only $3 \%$. For PLSVC patients, the hemodynamics were not abnormal, the clinical symptoms were not visible, and no treatment was needed. Previous studies showed that PLSVC could cause coronary sinus dilatation, and muscle fiber disorder of atrial ostium of the coronary sinus provides conditions for reentry and thus triggering supraventricular tachycardia [Ince 2007; Gu 2001]. Such uncommon vascular malformations attract extensive attention in cardiac surgery. Although the patient in the present study showed such kind of vascular malformations, no related arrhythmia was observed during the perioperative period. In this case, the aberrant bilateral subclavian artery originated from the aortic arch; however, this anomaly was not seen from the preoperative CTA, which may be due to the occlusion of the aortic arch aneurysm. After the intraoperative exploration, active treatment was performed. The open repair of aortic arch of the bilateral subclavian artery was conducted. In the meantime, the branches of the artificial blood vessels were anastomosed to the bilateral radial artery to maintain the regular blood supply of the upper limbs. After the operation, the upper limb blood pressure of the patient was maintained at $100 \mathrm{mmHg}-120 \mathrm{mmHg}$ with a rhythmic pulsation of bilateral radial arteries, and the movement and sensation of both upper limbs were all normal.

One of the most common symptoms of aortic aneurysm is bosom frowsty and chest pain caused by the enlargement of tumors and the compression of adjacent organs. Longduration compression can lead to thinning of the tracheal wall and destruction of the tracheal cartilage, eventually resulting in the softening of the trachea. In this study, the patient had apparent symptoms of tracheal compression and CT manifestations preoperatively. Postoperative signs included airway spasm and tracheal stenosis, due to tumor compression. After tracheal stent placement, the trachea cannula successfully was removed. The main indication for tracheal stenting is unresectable malignant tumor and benign lesions, such as tracheal stenosis and tracheal fistula, which cannot tolerate open surgery. Therefore, for such kinds of lesions, it is also appropriate to choose tracheal stenting because there is a risk of open trauma and tracheal softening if open surgery is used to relieve tracheal compression.

This case reported that for descending aortic aneurysm combined with vascular malformation and tracheal compression, total aortic arch replacement, elephant trunk surgery, and second-stage tracheal stent surgery are effective, thus providing feasible treatment options for patients with complex aortic lesions. Preoperative imaging examinations, intraoperative exploration of vascular variation, and restoring the hemodynamic stability as much as possible are necessary. However, when associated with other complications occurs, risks and benefits should be evaluated, and the timing of staging surgery at the right time also should be discussed during treatment. 


\section{REFERENCES}

Arima H, Sobue K, Tanaka S et al. 2002. Airway obstruction associated with transesophageal echocardiography in a patient with a giant aortic pseudoaneurysm. Anesth Analg. 95:558-60, table of contents.

Bickerstaff LK, Pairolero PC, Hollier LH et al. 1982. Thoracic aortic aneurysms: a population-based study. Surgery. 92:1103-8.

Borst HG, Walterbusch G, Schaps D. 1983. Extensive aortic replacement using "elephant trunk" prosthesis. Thorac Cardiovasc Surg. 31:37-40.

Chau KH, Elefteriades JA. 2013. Natural history of thoracic aortic aneurysms: size matters, plus moving beyond size. Prog Cardiovasc Dis. 56:74-80.

Chokr MO, de Moura LG, Aiello VD et al. 2018. Atrioventricular nodal reentrant tachycardia and persistent left superior vena cava: A tough nut to crack. Successful ablation with transseptal approach. HeartRhythm Case Rep. 4:589-93.

Crawford ES, Crawford JL, Stowe CL et al. 1984. Total aortic replacement for chronic aortic dissection occurring in patients with and without Marfan's syndrome. Ann Surg. 199:358-62.

Crawford ES, Svensson LG, Coselli JS et al. 1989. Surgical treatment of aneurysm and/or dissection of the ascending aorta, transverse aortic arch, and ascending aorta and transverse aortic arch. Factors influencing survival in 717 patients. J Thorac Cardiovasc Surg. 98:65973; discussion 73-4.

Gu X, Li S, Zhao F et al. 2001. Pacemaker leads were implanted in patients with persistent left superior vena cava and absent right superior vena cava.

Ince H, Nienaber CA. 2007. Etiology, pathogenesis and management of thoracic aortic aneurysm. Nat Clin Pract Cardiovasc Med. 4:418-27.

Kato M, Ohnishi K, Kaneko M et al. 1996. New graft-implanting method for thoracic aortic aneurysm or dissection with a stented graft. Circulation. 94:Ii188-93.

Kato M, Kuratani T, Kaneko M et al. 2002. The results of total arch graft implantation with open stent-graft placement for type A aortic dissection. J Thorac Cardiovasc Surg. 124:531-40.

Khalil A, Helmy T, Porembka DT. 2007. Aortic pathology: aortic trauma, debris, dissection, and aneurysm. Crit Care Med. 35:S392-400.

Kim T, Martin TD, Lee WA et al. 2009. Evolution in the management of the total thoracic aorta. J Thorac Cardiovasc Surg. 137:627-34.

Sabra O, Sabri A, Sfeir P. 2006. Airway obstruction secondary to thoracic aortic aneurysm leak. A case report. Eur Arch Otorhinolaryngol. 263:1144-6.

Xiong J, Zhang M, Guo W et al. 2013. Early malperfusion, ischemia reperfusion injury, and respiratory failure in acute complicated type B aortic dissection after thoracic endovascular repair. J Cardiothorac Surg. 8:17. 